\title{
Einleitung: KI ohne Grenzen?
}

\author{
Peter Gabriel
}

Der Phantasie, Anwendungen der KI zu ersinnen, sind keine Grenzen gesetzt: Maschinen und Software werden zu "intelligenten" Artefakten, die mühelos auch schwierigste Situationen meistern. Das reicht vom Chatbot, der in medizinischen Fragen eigenständig berät, bis hin zum autonomen Auto, das seine Fahrgäste sicher von A nach B bringt - auch im dichtesten Stadtverkehr. Aber in welchen Wirtschaftszweigen und in welchen Anwendungsfeldern wird KI tatsächlich in den kommenden Jahren ihr Potenzial, auch anspruchsvolle menschliche Tätigkeiten zu automatisieren, entfalten können? Zwar gibt es spektakuläre Einzelbeispiele, wie das Schachprogramm, das den Weltmeister schlägt, oder den Sprachassistenten im Smartphone. Darüber hinaus ist diese Frage aber nicht leicht zu beantworten, denn die meisten bekannten KIAnwendungen stecken in der Praxis noch in den Kinderschuhen.

Carl Benedikt Frey and Michael A. Osborne von der Universität Oxford hatten sich in ihrer viel zitierten und oft kritisierten Studie aus dem Jahr 2013 zur Auswirkung der „Computerisation" auf Jobs in den USA noch auf die allgemeine Analyse von Arbeitsplatzprofilen gestützt. Den Einsatz von KI und KI-gestützter Robotik erwarteten sie vor allem im Transportgewerbe und der Logistik, im produzierenden Gewerbe und im Dienstleistungssektor (Frey und Osborne 2013).

Mittlerweile gibt es aber auch erste empirische Studien, in denen sich Unternehmen sowohl zum Status quo von KI-Anwendungen als auch zu den Zukunftserwartungen an diese Technologie äußern.

Um solche Studien einzuschätzen, ist es lohnend, einen genauen Blick in die Betriebe selbst zu werfen und deren interne Abläufe - Beschaffung, Forschung und Entwicklung, IT, Personal, Finanzen, Logistik, Produktion/Betriebsführung, Marketing/Vertrieb, Kundendienst - zu betrachten. Das Beratungshaus Sopra Steria ist 2017 in einer Unternehmensbefragung so vorgegangen. Danach setzen die interviewten Unternehmen heute KI noch vor allem im IT-Betrieb sowie in der Produktion bzw. in ihren Standardprozessen ein. Hauptanwendung ist demnach die Automatisierung von einfachen, Software-basierten Routineprozessen, etwa der Eingabe einer Rechnung in eine Finanzbuchhaltung. Zunehmend automatisieren sie jedoch auch anspruchsvollere („intelligente“) Aufgaben der eigenen Produktion bzw. Dienstleistung. Das kann etwa eine automatische Erkennung fehlerhaft produzierter Bauteile mittels Bildanalyse sein oder die eigenständige Klassifikation von E-Mails in einem 
Callcenter, um etwa die Beschwerde zu einem Produkt von dem Wunsch nach Änderung der Lieferadresse zu unterscheiden.

Die Automatisierung von sehr einfachen Arbeitsprozessen bleibt laut Sopra Steria auch in Zukunft wichtig, allerdings wachsen die Ansprüche. An die erste Stelle der Zielvorstellungen rückt in den befragten Unternehmen die intelligente Automatisierung komplexerer Prozesse. Dem folgt der Wunsch nach digitalen Assistenten, die Mitarbeiter nicht ersetzen, aber sie in ihrer Tätigkeit unterstützen, etwa in Form eines sprachgesteuerten Hilfesystems für den Monteur in der Produktion. Zu diesem wachsenden Anspruch an die Leistungsfähigkeit der KI passt, dass in Zukunft KI-Technologien zunehmend auch den für den Unternehmenserfolg zentral wichtigen Kundendienst unterstützen sollen.

Für die meisten innerbetrieblichen Anwendungsbereiche läuft der Zweck des KI-Einsatzes vor allem auf eine Kostenreduktion hinaus. Aber nicht immer. Besonders in Marketing und Vertrieb sowie im Kundendienst erwarten die befragten Unternehmen, dass die Verwendung von KI-Innovationen in anderen Bereichen anstoßen kann. So will man mit gezielten Datenanalysen die Kunden und ihre Bedürfnisse besser verstehen und das eigene Angebot zielgerichtet darauf zuschneiden. Auch bei der Beschaffung gehen die befragten Unternehmen davon aus, dass sie mit KIbasierten Datenauswertungen die Qualität von Prozess und Ergebnis verbessern können (Sopra Steria 2017).

Da sich KI-Applikationen in den Unternehmen noch in einem sehr frühen Stadium befinden, wäre es noch zu voreilig, verlässliche Aussagen zum künftigen Einsatz dieser Technologie zu machen. Folgt man einer weltweiten Unternehmensbefragung des IT-Dienstleisters Infosys, liegen der Handel, die Konsumgüterindustrie, Telekommunikationsdienstleister und die Finanzwirtschaft an der Spitze der KI-Anwendungsbranchen. Die Tourismusindustrie und die öffentliche Verwaltung blieben demnach noch zurück (Infosys 2018).

Zu einem ähnlichen Ergebnis kommt auch eine volkswirtschaftliche Modellrechnung der Unternehmensberatung Accenture, die sich analog zu Frey und Osborne stark auf den potenziellen Automatisierungsgrad menschlicher Arbeit stützt. Danach können vor allem die Telekommunikationsdienstleister, das produzierende Gewerbe und die Finanzwirtschaft mit einer größeren Steigerung ihrer Bruttowertschöpfung durch $\mathrm{KI}$ rechnen. Auch nach dieser Modellrechnung ist die öffentliche Verwaltung das Schlusslicht (Purdy und Daugherty 2017).

Zu einer etwas anderen Einschätzung gelangt das kleine, erst 2014 gegründete Marktforschungsunternehmen Tractica: Demnach finden sich heute die wichtigsten $\mathrm{KI}$-Anwendungen bei den Internet- bzw. mobilen Diensten, seien es Sprachsteuerung oder die Kultivierung von Musik, Nachrichten und anderen digitalen Inhalten. 
Große Nachfrage sieht Tractica derzeit bei Rüstungsunternehmen, die automatisierte Waffen- und Logistiksysteme herstellen. Ein eher langfristig nutzbares Anwendungsfeld sehen die Meinungsforscher in der Dienstleistungsindustrie wie in der Finanzwirtschaft, der Medienindustrie, den unternehmensnahen Dienstleistungen und in der Gesundheitswirtschaft. Das besondere Potenzial der KI verorten sie vor allem bei der Verarbeitung von Sprach- und Bilddaten, wenn es darum geht, menschliche Tätigkeiten zu ersetzen oder zu unterstützen (Tractica 2016).

Bei aller Unsicherheit: In den folgenden Beiträgen werden die heute erkennbaren Ansätze der KI-Anwendung skizziert und gewinnen an Kontur: das autonome Auto, die Sprachübersetzung, die Robotik. Diese drei sind zurzeit wohl auch die Synonyme für KI in der öffentlichen Wahrnehmung, da die Technologie uns Menschen hier frappierend autark zur Seite tritt. Vom Gesundheitswesen erwarten viele Experten, dass es allein mit den KI-Verfahren des Maschinenlernens und der Bildanalyse noch gelingen kann, die immensen Datenmengen der digitalen Medizin effizient und sinnvoll auswerten zu können.

Bildung und öffentliche Verwaltung stehen noch nicht im Mittelpunkt der KI-Diskussion - zu Unrecht. Denn Digitale Bildung ist weit mehr als die Nutzung von Informations- und Kommunikationstechnik für die Vermittlung von Wissen. Lernen ist interaktiv und schließt ein ständiges Feedback mit ein. Die Erschließung der Nutzungsdaten digitaler Lernsysteme wäre hierbei äußerst hilfreich. KI-basierte Datenanalysen, die "Learning Analytics", können zu einem zentralen Element des Bildungssystems werden. Und ein womöglich noch größeres Potenzial zu unserer Unterstützung lässt sich mit KI-Systemen in der öffentlichen Verwaltung erschließen. In großer Zahl anfallende Standardvorgänge wie einfache Auskünfte und Leistungsberechnungen in der Sozialverwaltung lassen sich gut automatisieren - ohne dass die Qualität solcher staatlichen Dienstleistungen aus Sicht der Bürgerinnen und Bürger leiden müsste. 


\section{Literatur}

Purdy, M.; Daugherty, P. (2017): How Al Boosts Industry Profits and Innovation. Accenture.

Frey, C.; Osborne, M. A. (2013): The Future of Employment: How Susceptible are Jobs to Computerization? University of Oxford.

Infosys (2018): Leadership in the Age of Al. Adapting, Investing and Reskilling to Work Alongside Al.

Sopra Steria Consulting (2017): Potenzialanalyse Künstliche Intelligenz. Hamburg.

Tractica (2016): Artifical Intelligence. 10 Trends to Watch in 2017 and Beyond. Boulder, CO.

\section{(c) (1)}

Dieses Kapitel wird unter der Creative Commons Namensnennung 4.0 International Lizenz http://creativecommons.org/licenses/by/4.0/deed.de) veröffentlicht, welche die Nutzung, Vervielfältigung, Bearbeitung, Verbreitung und Wiedergabe in jeglichem Medium und Format erlaubt, sofern Sie den/die ursprünglichen Autor(en) und die Quelle ordnungsgemäß nennen, einen Link zur Creative Commons Lizenz beifügen und angeben, ob Änderungen vorgenommen wurden.

Die in diesem Kapitel enthaltenen Bilder und sonstiges Drittmaterial unterliegen ebenfalls der genannten Creative Commons Lizenz, sofern sich aus der Abbildungslegende nichts anderes ergibt. Sofern das betreffende Material nicht unter der genannten Creative Commons Lizenz steht und die betreffende Handlung nicht nach gesetzlichen Vorschriften erlaubt ist, ist für die oben aufgeführten Weiterverwendungen des Materials die Einwilligung des jeweiligen Rechteinhabers einzuholen. 